\title{
Modelo de simulación para determinar niveles de contaminación del Río Mantaro
}

\section{Simulation model to determine levels of contamination of the Mantaro River}

\section{RESUMEN}

Objetivos: Determinar si la contaminación del río Mantaro tiene alguna influencia sobre la viabilidad del programa "Sierra Exportadora"; luego de la construcción del modelo, se conducirá experimentos sobre el modelo con el fin de reducir la incertidumbre acerca de la influencia de la contaminación del río Mantaro sobre la viabilidad del programa "Sierra Exportadora" y evaluar los posibles resultados. Métodos: Construir un modelo de simulación donde se utilizará este sistema, el cual será útil para extrapolar los resultados posibles, dicha solución obtenida enmarcará los hitos más importantes que intervienen en el proceso; éstos elementos básicos del proceso están unidos entre sí, mediante flechas que indican las influencias que se establecen entre ellos; Además el diagrama da a conocer que el proceso posee una estructura con un bucle de realimentación negativa, la influencia constituye una descripción del proceso que se está estudiando. Después de haber realizado una simulación 50 veces del modelo con los distintos valores de entrada tales como la contaminación, la influencia de la población, la viabilidad de la oca y alcachofa, estos datos se almacenaron en las tablas generadas por el programa STELLA y luego estos datos fueron exportados a Excel para su análisis. Resultados: La construcción de este modelo de simulación reduce únicamente la incertidumbre provocada por uno de varios factores, la contaminación del río Mantaro sobre la viabilidad del Programa Sierra Exportadora. Conclusiones: La información generada por la investigación puede ser aplicada en otros sistemas que tengan características similares. Lo que propone precisamente es aportar e integrar todos los aspectos en un modelo general de simulación para el análisis de la viabilidad del Programa Sierra Exportadora.

Palabras clave: Simulación, influencia, contaminación, rio Mantaro.

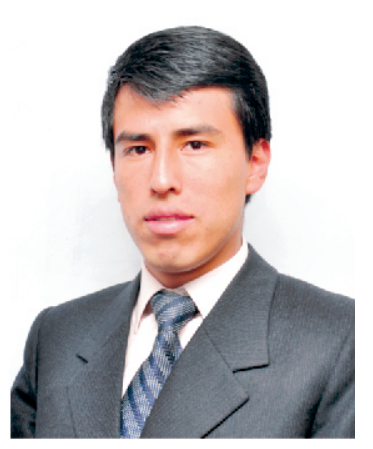

Iván Augusto Castillo Ponce Universidad Nacional de Huancavelica

\section{ABSTRACT}

Objectives: To determine if the contamination in the Mantaro river has some influence about the viability of the "Sierra Exportadora" program; after the construction of the pattern, will be conducting experiments in the model in order to reduce the uncertainty about the influence of the pollution of the Mantaro River on the viability of "Sierra Exportadora" program and to evaluate the potential results. Methods: Making a simulation model where this system will be used, which will be useful to extrapolate the possible results, this obtained solution will frame the most important landmarks that intervene in the process; these basic elements of the process are linked to each other. Besides, the diagram discloses that the process has a structure with a negative

1 Ing. en Informática, Docente de la Universidad Nacional de Huancavelica. 
feedback loops, the influence is a description of the process which is being studied. After the simulation was done 50 times in the model with different values of entrance values such as the contamination, influence of population, the viability of the goose and artichoke, these data were stored in the generated charts by the Stella software and then these data were exported to Excel software for their analysis. Results: The construction of this simulation model only reduces the uncertainly caused by one of several factors, the contamination of the Mantaro River about the viability of the "Sierra Exportadora" program. Conclusions: The information generated by the investigation can be applied in other systems that have similar characteristic. What is proposed in fact to contribute and to integrate all the aspects in a general simulation model for the viability of the "Sierra Exportadora" program.

Key words: simulation, influence, pollution, Mantaro River.

\section{INTRODUCCIÓN}

El Programa denominado "Sierra Exportadora", cuyos objetivos son fortalecer y hacer competente al productor de la sierra para que sus productos sean exitosamente exportables. El Valle del Mantaro es una de las zonas que forma parte de este programa; en dicho valle, una de las principales fuentes de irrigación es el río Mantaro, río contaminado por muchos años. Si los productos de la sierra van a ser exportados probablemente no pasaran los estándares de calidad que los países extranjeros exigen.

Existen estudios previos acerca de la contaminación del río Mantaro hechos por diversas instituciones. El trabajo tomó en cuenta las siguientes investigaciones: 1) Tesis doctoral: "Integración de modelos de simulación en el diseño de los ensayos clínicos", realizado por Ismai Abbas. Barcelona, Diciembre de 2003. 2) Los Modelos de Simulación Computacional de Incendios Universidad de Cantabria. Santander, Julio 2004. 3) Simulación numérica de la dinámica del agua en el suelo. Aplicación al diseño de sistemas de riego LAF. Francisco Ramírez de Cartagena Bisbe. Universitat de Lleida. Salamanca, 1999.

El problema identificado es, determinar la influencia de la contaminación del río Mantaro sobre la viabilidad del Programa Sierra Exportadora.

Se puede determinar la influencia de la contaminación del río Mantaro sobre la viabilidad del programa "Sierra Exportadora", mediante la construcción de un modelo de simulación.

El objetivo es determinar si la contaminación del río Mantaro tendría alguna influencia sobre la viabilidad del programa "Sierra Exportadora".

Es necesaria la construcción de un modelo de simulación sobre la influencia de la contaminación del rio Mantaro en el programa "Sierra Exportadora" con el propósito de predecir y reducir la incertidumbre.

En cuanto a las limitaciones se puede mencionar que el estudio de la contaminación del río Mantaro, únicamente se considera la contaminación por metales; para tal fin, se cuenta con datos recopilados desde el año 2000. Por consiguiente se investigará la influencia que ocasiona la contaminación del río Mantaro sobre la viabilidad del programa "Sierra Exportadora".

1) La variedad de disciplinas necesarias para abordar los objetivos de la tesis han hecho muy difícil identificar y aplicar los métodos, lo que hace que el sistema estudiado sea muy complejo 2) La limitación de la tesis es el no haber podido desarrollar un sub modelo más completo acerca de la influencia que tendría la población de la región Junín sobre la contaminación del río Mantaro. 3) El modelo construido únicamente está elaborado para una simulación en el lapso de tiempo de 2007 al 2011, y no se podrá extender el intervalo de tiempo mencionado.

Lo que propone en esta investigación como principal aportación es integrar todos los aspectos en un modelo general de simulación para el análisis de la viabilidad del Programa Sierra Exportadora, en particular en la región Junín. Y por lo cual si hay contaminación del río Mantaro, entonces esto afectara directamente a los sembríos, entonces el Programa Sierra Exportadora se vería directamente afectado. 




Figura 1: Diagrama de Influencias General

\section{MATERIAL Y MÉTODOS}

El proceso se puede representar en forma sintética mediante un diagrama de influencias. Este diagrama indican los hitos más importantes que intervienen en el proceso. Estos elementos básicos del proceso están unidos entre sí, mediante flechas que indican las influencias que se establecen entre ellos; cuando se haga referencia al "Valle del Mantaro". Este diagrama da a conocer que el proceso posee una estructura con un bucle de realimentación negativa, la influencia constituye una descripción del proceso que se está estudiando.

\section{Método}

En base al análisis y experiencia, la metodología para planificar un modelo válido y creíble debe de tener los pasos básicos. Según la Dinámica de sistemas el procedimiento de investigación a seguir es el siguiente:

- Definición del problema. Se debe reconocer sin ningún problema y analizando los términos de sistemas. Para ello el problema debe ser susceptible para el análisis en elementos y componentes.

- Conceptualización del sistema. Se trata de acometer definiendo los distintos elementos que integran, así como las influencias que se producen entre ellos.

- Formalización. Convertir el diagrama de influencias, alcanzado anteriormente, en el de Forrester. A partir de esto se pueden escribir las ecuaciones del modelo y posterior se hará un modelo del sistema programado en un computador.

- Comportamiento del modelo. Consiste en la simulación informática para determinar las trayectorias que genera.

- Evaluación del modelo. Se somete a una serie de ensayos y análisis para evaluar su validez y calidad.

- Explotación del modelo. Se emplea para analizar políticas alternativas que se está estudiando. 


\section{Programas utilizados}

El modelo implica hacer una simulación informática para ello se necesita la ayuda de un informático con el fin de traducir todos los componentes del modelo conceptual y que sea ejecutable para un ordenador, las transformaciones de estos se dará en tres formas: 1) utilizar lenguajes de programación generales (como el $\mathrm{C}++$ ), 2) utilizar herramientas de simulación, 3) utilizar programas específicos.

Un lenguaje de simulación permite reducir el tiempo de la programación y es mucho más fácil de manejar que un lenguaje de programación general. Para la construcción de nuestros modelos se ha optado por utilizar el software Stella, este programa responde a las necesidades de lo que se pretende programar.

\section{Stella}

El software STELLA es un programa de simulación por computadora, que proporciona una estructura e interfaz gráfica de fácil entendimiento, para observar la interacción cuantitativa de las variables dentro de un sistema. STELLA cuando se carga muestra la capa de "mapa/modelo", y esta capa muestra los diagramas del sistema en estudio a su diagrama de Forrester. El programa también genera ecuaciones y son la que gobierna toda la simulación. Además permite realizar automáticamente una serie de simulaciones cambiando el valor de uno o varios parámetros en cada simulación.

\section{Métodos para obtener la población}

La siguiente información muestra la evolución de la población en la región Junín.

Tabla1: Evolución de la población en la región Junín

\begin{tabular}{cccc} 
Año & Población Total & Incremento Anual & $\begin{array}{c}\text { Tasa de } \\
\text { Crecimiento } \\
\text { (Promedio anual) }\end{array}$ \\
\hline 1940 & 381343 & - & - \\
1962 & 546662 & 165319 & 1,7 \\
1972 & 720457 & 173795 & 2,6 \\
1981 & 896962 & 176505 & 2,5 \\
1993 & 1092993 & 196031 & 1,7 \\
1995 & 1140505 & 47512 & 1,4 \\
1996 & 1156059 & 15554 & 1,4 \\
1997 & 1171698 & 15639 & 1,4 \\
1998 & 1187279 & 15581 & 1,3 \\
1999 & 1202663 & 15384 & 1,3 \\
2000 & 1217708 & 15045 & 1,3 \\
2001 & 1232343 & 29680 & 1,2 \\
2002 & 1246663 & 28955 & 1,2 \\
2003 & 1260773 & 28430 & 1,1 \\
\hline & & & \\
\hline
\end{tabular}




\section{Aportes que mejoraron el procedimiento}

Todo modelo se construye para ayudar a resolver un problema concreto. Sin embargo, esa explotación puede tomar formas variadas, en primer lugar, permite hacer predicciones. Segundo las utilizaciones se incorporan una cierta imprecisión, para analizar las tendencias de evolución de esas magnitudes. Por último, consiste en emplearlos como instrumentos para analizar el comportamiento del sistema estudiado.

\section{Análisis estadísticos}

Para tener una información estadística sobre el comportamiento del sistema ante perturbaciones exteriores. Ahora el diagrama debe ser trasladado al diagrama de Forrester. Por ello se tomará en cuenta la observación de los distintos elementos que aparecen en los nodos de un diagrama de influencias, algunos representan variaciones con respecto al tiempo de otras magnitudes.

\section{RESULTADOS}

Para el análisis de sensibilidad del modelo, se procedió a simular 50 veces el modelo con los distintos valores de entrada, se almacenaron los datos generados en una tabla de STELLA y luego estos datos fueron exportados a Excel para su análisis. Observando los resultados, se puede concluir que el análisis de sensibilidad muestra que el modelo es sensible a la variable "contaminación", porque los índices de viabilidad cambian drásticamente al cambiar el valor asignado a la variable ya mencionada. Por otro lado se nota que el modelo es insensible a la variable "influencia", porque no se altera en nada los índices de viabilidad cuando cambia el valor asignado a la variable "influencia".

Por último se puede concluir que los resultados obtenidos mostraron claramente que la contaminación del río Mantaro si tiene influencia en el programa Sierra Exportadora.
La relación que se establece por las simulaciones del modelo, es que si hay contaminación del río Mantaro, y esta contaminación afecta directamente los sembríos, entonces el Programa Sierra Exportadora se vería directamente afectado.

\section{DISCUSIÓN}

La información generada por la investigación puede ser aplicada en otros sistemas que tengan características similares. Lo que propone precisamente es aportar e integrar todos los aspectos en un modelo general de simulación para el análisis de la viabilidad de PSE. Por lo tanto la construcción de este modelo reduce únicamente la incertidumbre provocada por uno de varios factores, la contaminación del río Mantaro. La relación que se establece es que si hay contaminación del río Mantaro, y esta contaminación afecta directamente a los sembríos, entonces el Programa Sierra Exportadora se vería directamente afectado. 


\section{REFERENCIAS BIBLIOGRAFICAS}

1. Abbas I. Integración de modelos de simulación en el diseño de los ensayos clínicos [Tesis para optar el grado de Doctor]. Barcelona; 2003. Universidad de Cataluña.

2. Annak C, Barandiaran A. La Oroya can not wait. Primera Edición. Lima: Asociación Interamericana para la Defensa del Ambiente AIDA; 2003. pp. 130.

3. García P. Sierra Exportadora Empleo, Modernidad y Justicia en los Andes. Biblioteca Nacional del Perú. Lima, Perú. 2005.

4. Caballero JM. Economía agraria de la sierra Peruana. Una estrategia del desarrollo rural para la sierra del Perú. Lima: IEP, Banco Mundial. 2002.

5. INEI. Estimaciones de la Población por Departamentos, Provincias y Distritos 1995 2005. Censos Nacionales de 1940, 1961, 1972, 1981 y 1993. 2005.

6. Universidad San Ignacio de Loyola. Manual para la formulación y evaluación de proyectos de inversión Lima (editor), 2000. 120 pp.

7. Actualidad Minera Del Peru, Evaluación de la Calidad de Aire, Agua y Suelos en la Provincia de Yauli La Oroya: Una experiencia participativa. Perú 2000, № 19. 15 pp.

Correo electrónico:

iacastillop@hotmail.com 